\title{
Drag on a Flat Plate in Low-Reynolds-Number Gas Flows
}

\author{
Quanhua Sun* and Iain D. Boyd ${ }^{\dagger}$ \\ University of Michigan, Ann Arbor, Michigan 48109
}

\begin{abstract}
Airflow over a flat plate at zero incidence is investigated as a function of the Reynolds number $R e$ and the Mach number $M$ under subsonic, low-Reynolds-number conditions. The flows are simulated using the direct simulation Monte Carlo (DSMC) method and the information preservation (IP) method that is a modified DSMC method developed for low-speed rarefied gas flows. Good agreement is obtained between the DSMC and IP results and between the IP results and available experimental data. The simulations predict that the drag coefficient on the flat plate depends on the Reynolds number and the Mach number, and both the rarefied and compressible effects on the drag coefficient increase when the flow Reynolds number decreases. It is found that the normalized drag $C_{D} \cdot M$ depends on $\sqrt{ }(R e) / M^{0.8}$ when this parameter varies between 1 and 100 , which suggests a scaling law for engineering analysis.
\end{abstract}

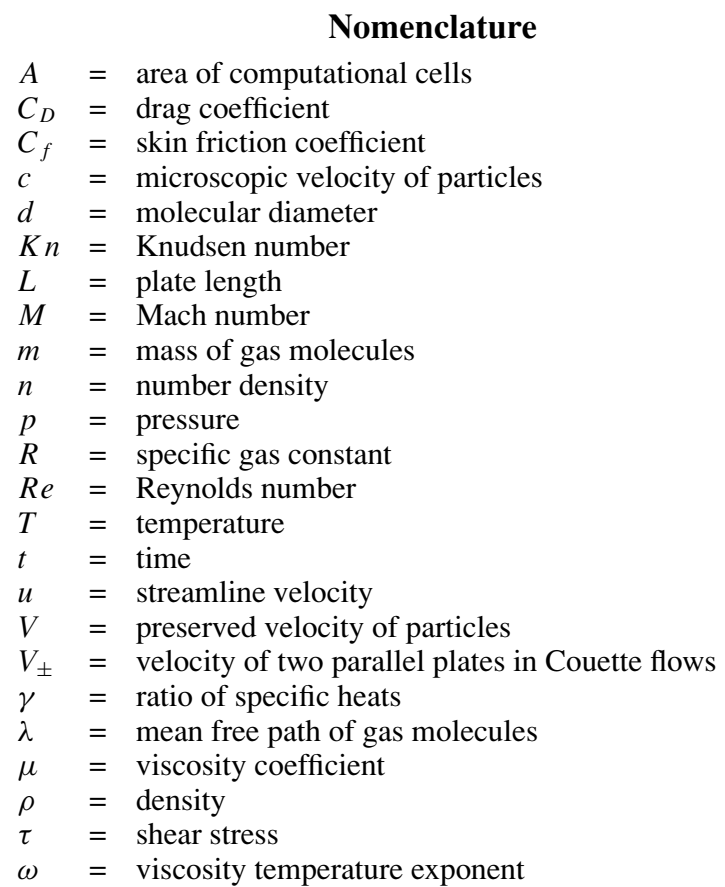

Subscripts

in $=$ incident particles

re $=$ reflected particles

$x=$ at $x$ location

$0=$ reference condition

$\infty=$ freestream condition

\section{Introduction}

T HE problem of flow past a flat plate at zero incidence is one of fundamental interest in fluid mechanics because it generates a wide range of basic flow phenomena. At a high-Reynolds-

Received 25 July 2003; revision received 24 November 2003; accepted for publication 8 March 2004. Copyright (C) 2004 by the American Institute of Aeronautics and Astronautics, Inc. All rights reserved. Copies of this paper may be made for personal or internal use, on condition that the copier pay the $\$ 10.00$ per-copy fee to the Copyright Clearance Center, Inc., 222 Rosewood Drive, Danvers, MA 01923; include the code 0001-1452/04 \$10.00 in correspondence with the CCC.

${ }^{*}$ Research Fellow, Department of Aerospace Engineering. Member AIAA.

${ }^{\dagger}$ Professor, Department of Aerospace Engineering. Associate Fellow AIAA. number condition, the flow experiences the leading-edge, laminar boundary-layer, turbulent transition, turbulent boundary-layer, trailing-edge, and wake regions. Furthermore, the flow is rarefied within the leading- and trailing-edge regions with a scale of several mean free paths of the gas molecules. The rarefied effects, however, are negligible because the rarefied domain is so small compared to the plate length scale. Therefore, the Navier-Stokes (NS) equations (or turbulent model equations) can describe high-Reynolds-number flows, and even the boundary-layer equations can predict the drag on a flat plate. However, when the flow Reynolds number decreases, the interaction between the boundary layer and the outer stream becomes strong, which results in larger leading- and trailing-edge regions. Meanwhile, low-Reynolds-number flows generally mean that the rarefied effects (e.g., the slip velocity of gas on the plate) are important, because the Reynolds number is inversely proportional to the Knudsen number if the Mach number remains unchanged $(R e=M / K n)$. Then the NS equations or their approximations are not valid any more, and kinetic theory must be applied to study low-Reynolds-number flows.

Flow past a flat plate has been studied extensively for incompressible flows at low Reynolds numbers. ${ }^{1}$ Studies focused on solving continuum equations with a slip velocity boundary condition. Schaaf ${ }^{2}$ considered a linearized slip flow past a semi-infinite flat plate using a boundary-layer form of the Oseen equations. Laurmann $^{3}$ studied the same problem but used the full linearized Oseen equations, whereas Murray ${ }^{4}$ obtained an asymptotic solution to the Navier-Stokes equations. When the mean free path $\lambda$ of the gas is small (strictly speaking, when the Knudsen number based on the boundary-layer thickness is small), the correction for the shear stress to the Blasius solution is $O\left(\lambda^{2}\right)$ according to Schaaf, $O(\lambda)$ according to Laurmann, and $O\left[\log \lambda / R e_{x}\right]$ according to Murray. However, these methods are more or less approximate, and comparison between their results and experimental data is difficult because phenomena with a length scale of several mean free paths are difficult to detect in an experiment with a "semi-infinite" flat plate. On the other hand, the NS equations were solved with a no-slip velocity boundary condition for flow over a plate having a finite length. Janssen ${ }^{5}$ obtained a solution using an electrical analog method. However, Janssen's results are unsatisfactory because not enough lattice points were used. Later, Dennis and Dunwoody ${ }^{6}$ obtained much better results using a numerical approach. The drag predicted by Dennis and Dunwoody agrees very well with experimental results of Janour ${ }^{7}$ for Reynolds numbers between 10 and 1000 .

Compressible flow past a flat plate is much more complicated. For supersonic, low-Reynolds-number flows, slip effects are masked by shock wave-boundary layer interactions. There is also strong nonequilibrium among internal energy modes. To exclude the shock wave effects, we limit our discussion to subsonic flows. At highReynolds-number flow condition, the Blasius solution is still valid 
and the Reynolds number is the dominant parameter. However, for low-Reynolds-number flows, the Mach number becomes another important parameter even when the flow speed is small as indicated in the experiments by Schaaf and Sherman. ${ }^{8}$ Moreover, the rarefied effects are not limited to the slip velocity. Schaaf and Sherman pointed out that the higher order approximations to the stress tensor and heat flux vector than those given by the Navier-Stokes expressions must also be considered. Hence, approaches based on kinetic theory may be needed to accurately predict the drag on a flat plate in low-Reynolds-number compressible flows.

In this paper, we simulate subsonic, low-Reynolds-number gas flows past a flat plate at zero incidence using two particle approaches: the direct simulation Monte Carlo (DSMC) method and the information preservation (IP) method. We first discuss several numerical difficulties and summarize the information preservation method in Sec. II. Then the general numerical procedure is described in Sec. III. Numerical results and discussions are presented in Sec. IV, where the correlation of the drag on the flat plate is investigated. Finally, we end with some concluding remarks.

\section{Numerical Approach}

For compressible gas flow at low-Reynolds-number condition, it is clear that the no-slip boundary condition is invalid, and it is very possible that the Navier-Stokes equations are inappropriate because the shear stress and heat flux may not be modeled correctly. Even in cases where the NS equations are valid, there are some factors limiting the application of numerical methods based on the NS equations. First, there is no generally applicable criterion to indicate when the NS equations break down although there are several suggested continuum breakdown parameters. Second, current slip models are based on a one-dimensional Knudsen layer approximation. This approximation may be invalid near sharp edges, corners, and other singular points on a body. Kogan ${ }^{9}$ pointed out that the drag on a plate at zero incidence cannot be predicted by solving the NS equations with a general slip boundary condition to an accuracy of order $1 / R e$. Therefore, the most generally valid numerical approaches are those based on kinetic theory.

There are several popular methods based on kinetic theory, including direct Boltzmann methods ${ }^{10}$ and molecular dynamics techniques. ${ }^{11}$ The main difficulty of these methods is the tremendous numerical cost, which limits their application to simple geometry problems. The DSMC method, ${ }^{12}$ on the other hand, is very effective for high-speed rarefied gas flows. This method decouples the particle motions and interparticle collisions over small time intervals in simulated physical space where each particle represents a large number of real gas molecules. Particle motions are modeled deterministically, whereas the collisions are treated statistically. Using these strategies, the DSMC method is several orders of magnitude more numerically efficient than the molecular dynamics simulation. Therefore, the DSMC method has been widely used in many fields, including rarefied atmospheric gas dynamics, materials processing, and vacuum systems. However, it is a challenge to apply the DSMC method for simulating low-speed flows. First, the statistical noise associated with the DSMC method makes its results difficult to interpret for low-speed gas flows unless a huge sampling size is used. Second, conventional boundary conditions for the DSMC method, such as freestream conditions and vacuum conditions, do not work for subsonic flows. Instead, characteristic boundary conditions are required to capture the propagation of flow information across the boundaries.

To reduce the statistical difficulty associated with the DSMC method, an IP technique ${ }^{13}$ was proposed to extend the application of the DSMC method to low-speed gas flows. ${ }^{14,15}$ The IP method simulates microscopic particles as in the DSMC method but also solves information at the macroscopic level to obtain macroscopic flow information. Namely, a large number of represented particles are simulated in the computational domain, and each simulated particle is assigned with microscopic information (spatial coordinate, microscopic velocity, internal energy, etc.) and preserved information at the macroscopic level (flow velocity, temperature). During the simulation, every particle moves according to its microscopic velocity and experiences collisions with other particles and walls as in the DSMC method. The preserved information, however, is modeled based on the transfer equation of the Boltzmann equation. For example, the transfer equation for momentum can be written as Eq. (1), and the preserved velocity of particle $i$ is modeled according to Eq. (2):

$$
\begin{aligned}
& \frac{\partial}{\partial t}\left(n m \overline{\boldsymbol{V}_{i}}\right)+\nabla \cdot\left(n m \overline{\boldsymbol{c}_{i} \boldsymbol{V}_{i}}\right)=-\nabla \cdot\left[n m \overline{\left(\boldsymbol{c}_{i}-\boldsymbol{V}_{i}\right)\left(\boldsymbol{c}_{i}-\boldsymbol{c}_{0}\right)}\right] \\
& \quad=-\nabla p^{\prime}+\nabla \cdot \boldsymbol{\tau}^{\prime} \\
& \frac{\partial}{\partial t}\left(n m \boldsymbol{V}_{i}\right)+\text { microscopic movement }=-\nabla p+\text { collisions }
\end{aligned}
$$

where $\boldsymbol{V}_{i}$ is the preserved velocity of particle $i ; \boldsymbol{c}_{0}$ is the flow velocity; $\boldsymbol{c}_{i}$ is the microscopic velocity of particle $i ; n$ is the number density; $m$ is the mass of gas molecules; and $p^{\prime}$ and $\tau^{\prime}$ are the normal and shear stresses of the collision term in the transfer equation, respectively. In other words, the preserved information at the macroscopic level is initialized by the ambient flow condition or the inflow/outflow boundary condition, is carried by the particle, is updated during collisions, and is modified to include the pressure effects. The cell information is then set as the average of the corresponding preserved information of particles in the cell, and the density is obtained by solving the continuity equation using a finite volume method. Finally, the simulation results are obtained by sampling the cell information after the simulation reaches a steady state. It should be mentioned that the DSMC results are also available in an IP simulation. A flowchart of the DSMC-IP method is shown in Fig. 1.

The IP method has been applied to simulate many challenging problems, ${ }^{16-18}$ and very good results have been obtained. In general,

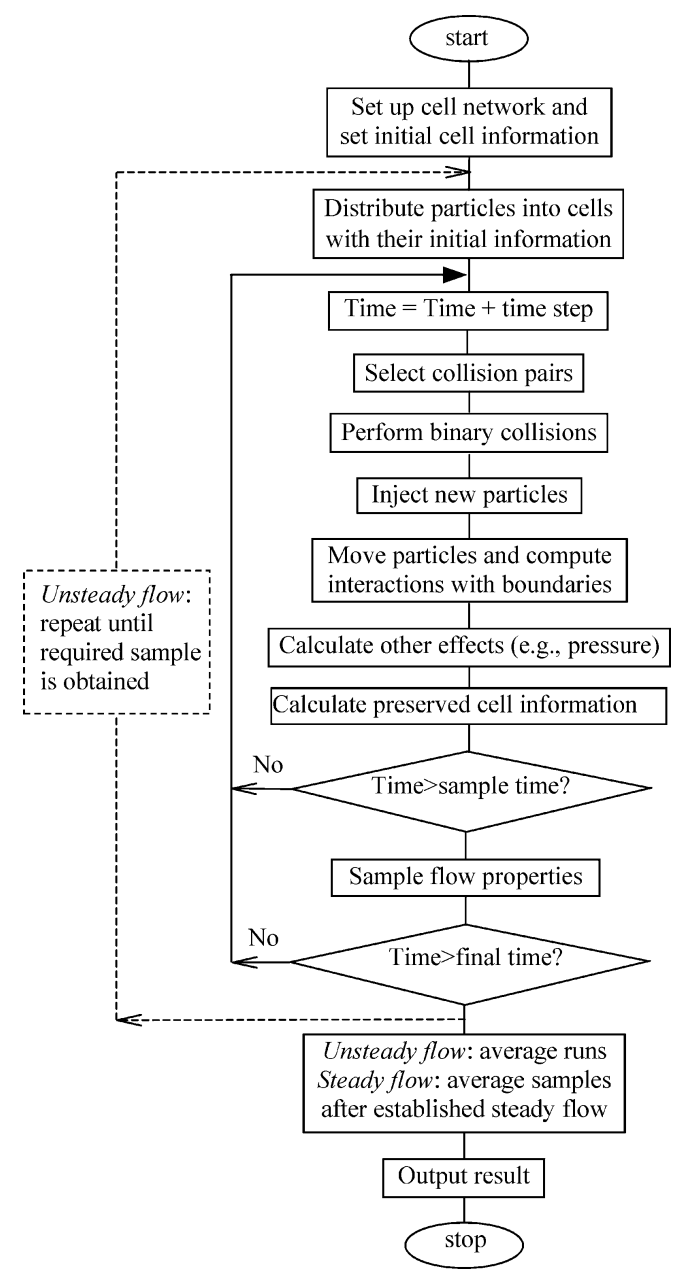

Fig. 1 DSMC-IP flowchart. 
Table 1 Statistical scatter associated with the IP and DSMC methods obtained when simulating Couette flows

\begin{tabular}{lclc}
\hline \hline \multirow{2}{*}{$\begin{array}{l}\text { Flow condition } \\
(K n=0.01)\end{array}$} & \multirow{2}{*}{$\begin{array}{c}\text { Sample size, } \\
\text { particles/cell }\end{array}$} & \multicolumn{2}{c}{ Statistical scatter, $\mathrm{m} / \mathrm{s}$} \\
\cline { 3 - 4 }$V_{ \pm}= \pm 100 \mathrm{~m} / \mathrm{s}$ & 1,000 & 0.6631 & 11.74 \\
& 10,000 & 0.5864 & 6.284 \\
$V_{ \pm}= \pm 1 \mathrm{~m} / \mathrm{s}$ & 100,000 & 0.2572 & 3.075 \\
& 1,000 & 0.006448 & 11.35 \\
& 10,000 & 0.006916 & 5.820 \\
$V_{ \pm}= \pm 0.01 \mathrm{~m} / \mathrm{s}$ & 100,000 & 0.002422 & 3.750 \\
& 1,000 & 0.000046 & 10.33 \\
& 10,000 & 0.000065 & 6.208 \\
& 100,000 & 0.000023 & 3.433 \\
\hline \hline
\end{tabular}

the validity of the IP method depends on whether the shear stress and the heat transfer are correctly simulated for a flow. For low-speed gas flows, it seems that the phenomenological collision and energy flux models ${ }^{15}$ work very well. However, for high-speed flows, a new energy flux model ${ }^{19}$ is required. It is also necessary to preserve temperatures in different modes (translational components, rotational and vibrational) for strongly nonequilibrium flows.

The main benefit of the IP method is its relatively small statistical scatter compared with the DSMC method for modeling low-speed flows, which can greatly reduce the computational cost for these flows. This is because the statistical scatter of the DSMC method arises directly from the thermal movement of particles whereas the thermal movement of particles causes scatter for the preserved information only at the macroscopic level. The statistical scatter associated with the IP and DSMC methods can be illustrated by Couette flows. The Couette flows have opposite velocities $\left(V_{ \pm}\right)$for the parallel plates, and argon gas is used. The velocity scatter obtained in the middle of the flows is listed in Table 1, where the data are obtained by averaging 100 samples. The results show that the scatter associated with the DSMC method is independent of the flow velocity whereas the scatter associated with the IP method decreases when the flow velocity drops. Hence, the IP method is good to simulate low-speed flows for which the DSMC method is numerically too expensive to control the statistical scatter.

Another benefit of the IP method is to implement effective boundary conditions for particle methods. Because the IP method has flow information preserved in computational cells with small statistical scatter, traditional boundary treatments used in computational fluid dynamics, (e.g., the characteristic boundary condition) can be used for the IP method. The DSMC method, embedded in the IP method, can therefore use the same boundary condition, which circumvents some difficulties in implementing boundary conditions for the DSMC method. ${ }^{20}$ The preserved cell information in the IP method can also help develop hybrid continuum/particle approaches. ${ }^{21}$

Therefore, to predict drag on a flat plate in low-Reynolds-number gas flows, the DSMC and IP methods are applied to simulate the flow using characteristic boundary conditions.

\section{Numerical Procedure}

The DSMC method and the IP method are applied to study flow over a flat plate at several flow Mach number and Reynolds number conditions. The flat plate has a finite length of $30 \mu \mathrm{m}$. The temperature of the plate is fixed at $295 \mathrm{~K}$ during simulation and full momentum and thermal accommodation is assumed. The freestream is air with a temperature of $295 \mathrm{~K}$, while the Mach number varies from 0.2 to 0.8 and the plate Reynolds number is between 0.2 and 100 . Because it is not necessary to mention all numerical specifications for all cases, we describe the general procedure for the simulations.

The freestream condition is specified by the Mach number and the Reynolds number. Namely, the streamline velocity is determined as

$$
u_{\infty}=M \cdot \sqrt{\gamma R T_{\infty}}
$$

The freestream density can be expressed as

$$
\rho_{\infty}=\left(R e \cdot \mu_{\infty}\right) / u_{\infty} L
$$

where $\mu_{\infty}$ is the viscosity coefficient of air at the freestream temperature, which is calculated using a simple temperature-law model:

$$
\mu_{\infty}=\mu_{0}\left(T_{\infty} / T_{0}\right)^{\omega}
$$

Here, $\mu_{0}=1.716 \times 10^{-5} \mathrm{~N} \cdot \mathrm{s} / \mathrm{m}^{2}$, the reference viscosity coefficient at $T_{0}=273 \mathrm{~K}$; and $\omega=0.77$, the viscosity temperature exponent. The global Knudsen number based on the plate length can also be obtained as

$$
K n=\lambda / L=1 /\left[\sqrt{2} \pi d_{0}^{2} n\left(T_{0} / T\right)^{\omega-\frac{1}{2}} \cdot L\right]
$$

or approximately as

$$
K n=1.19(M / R e)
$$

The computational domain is finite because it has to be for the simulation. Hence, appropriate boundary conditions are required. Far-field boundary conditions, however, cannot be directly applied on the boundaries, and so characteristic boundary conditions ${ }^{22}$ are used. It is assumed that the gradients of pressure and velocity along the flow boundary are negligible. For subsonic flow, the stagnation pressure and temperature are specified for the inflow boundary and the exit pressure is given for the outflow boundary. The size of the computational domain is tested and determined so that the simulated drag on the plate is independent of the domain size using the characteristic boundary condition. The domain has a radius of 3.515.5 plates in length for all cases considered. Although the smallest domain may seem rather small, it is found that the predicted drag on the plate agrees well with results using a larger domain and with the experimental data.

The computational cell size is required to be less than the mean free path of air molecules for the DSMC and IP methods. However, the cell size can be increased where the gradients of flow properties are small. This argument is verified by comparing the DSMC-IP results with the solution obtained using a hybrid continuum/particle approach, ${ }^{21}$ where the size of the particle cells is always less than the mean free path of molecules for a typical case. Hence, the cell size is set to be less than the mean free path of air for computational cells near the plate, and it is much larger than the mean free path of air molecules for cells far away from the plate to reduce the computational cost. Half of a typical computational grid is shown in Fig. 2 where the computational cells are clustered near the plate. The effect of the cell size is tested using the subcell technique. ${ }^{12}$ This technique restrains the collision partner for a particle in the sense that a computational cell is divided into several subcells and only particles in the same subcell can have collisions. For all cases, 3000-10,000 computational cells are used, and 4 or 9 subcells are employed in each computational cell for cases where the cell size is not small enough. To avoid having large cells with many particles and small cells with few particles, a particle weighting scheme is used, which means that particles in one cell may represent more real gas molecules than other particles in another cell. The particle weight for a cell is generated based on the volume (or area) of the cell. The use of the weighting scheme, however, will increase the statistical scatter and the scatter can be decreased by increasing the sample size. On average, about 30-150 particles are used for

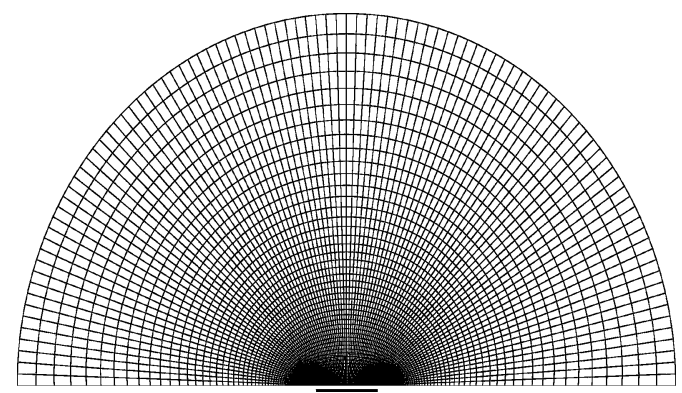

Fig. 2 Half of a typical computational grid for flows over a flat plate. 


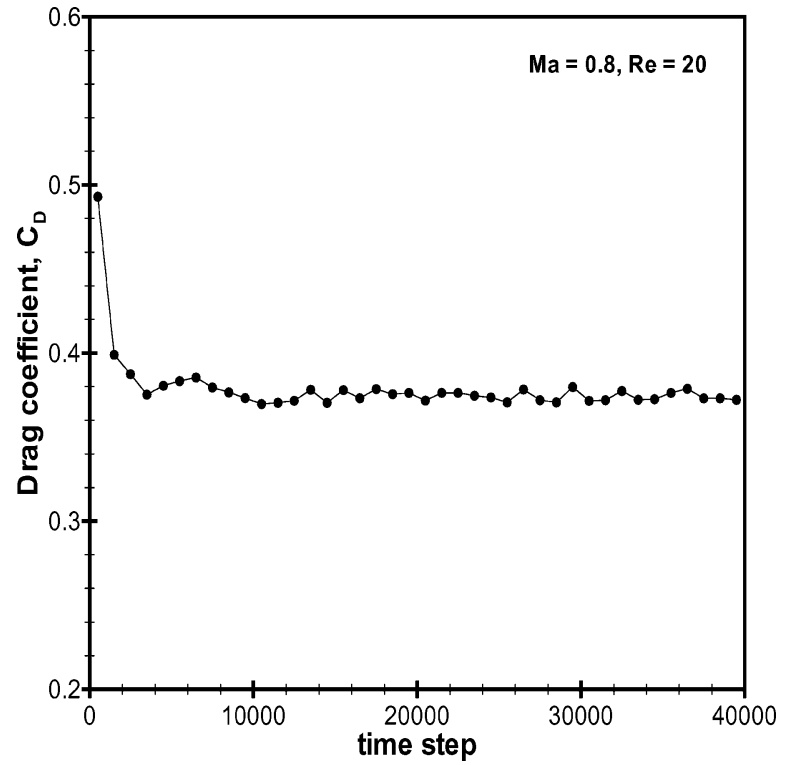

Fig. 3 History of the drag coefficient on the plate obtained over 1000 time steps with a sampling size of about 100,000 particles per cell.

each computational cell. (The larger number is used when the subcell technique is employed.) The time step is between $0.5 \times 10^{-10}$ and $5 \times 10^{-10} \mathrm{~s}$, which is less than the mean collision time of air molecules, another requirement of the DSMC and IP methods.

Both DSMC and IP methods are approaches for solving unsteady flow problems. Hence, steady results can only be obtained after the flow reaches a steady state. Figure 3 shows the history of the drag coefficient on the plate that is obtained using the IP method after sampling over 1000 time steps with a sampling size of about 100,000 particles per cell. For the case shown in this figure, the flow reaches a steady state after less than 20,000 time steps, while the fluctuation of the drag coefficient is due to the statistical scatter associated with the IP method. It is clear that good results can be obtained with a large sampling size. However, it is difficult to determine the statistical scatter associated with the DSMC and IP results because the sampled particles are not statistically independent. In general, a sample size of the order of $10^{7}$ particles per cell is used for the results given in the next section, and a simulation takes about 20 to $40 \mathrm{~h}$ on a Pentium 4 personal computer. It is estimated that the numerical error for the drag coefficient is less than 0.1 for the DSMC results and is less than 0.01 for the IP results.

\section{Numerical Results and Discussion}

Many results can be obtained using the DSMC method and the IP method when simulating flow over a flat plate, including flowfields and surface properties. In this paper, the overall drag on the plate is investigated under subsonic, low-Reynolds-number conditions. First, the drag obtained using the DSMC and IP methods is compared when the freestream Mach number is 0.2 and 0.8. Then the drag is calculated under several Mach number and Reynolds number conditions, which indicates that the drag coefficient depends on both the Mach and Reynolds numbers. Finally, the simulated results are compared with available experimental data, and a scaling law for the drag coefficient under simulated flow conditions is proposed.

When a freestream flows over the flat plate, the flow is slowed down due to collisions between gas molecules and the plate surface, which generates the drag on the plate. On the other hand, because the flow is slowed down, the gas is compressed as it faces the plate and is expanded as it leaves the plate. Figures 4-6 show the density and velocity contours under three flow conditions, which illustrate the dependence of the flowfields on the Reynolds and Mach numbers. [Knudsen number effects can be obtained from these effects because they are directly related; see Eq. (7).] In general, the flow is more compressible when the Reynolds number is smaller or the Mach number is larger. For example, in Fig. 4, there is only a

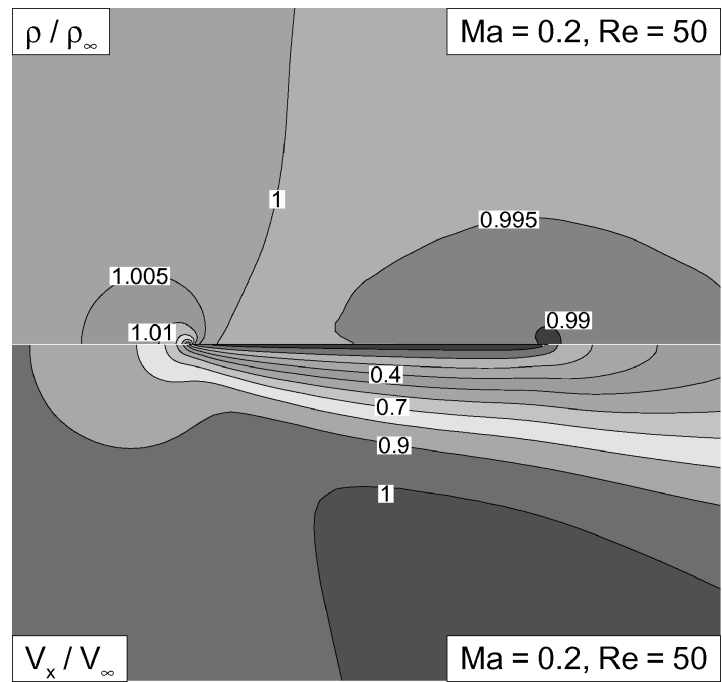

Fig. 4 Density and velocity contours when $M=0.2$ and $R e=50$.

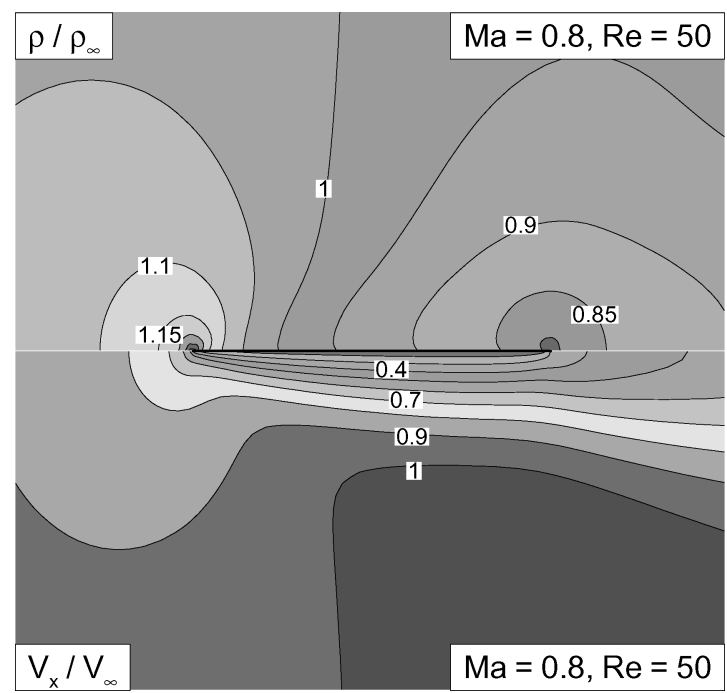

Fig. 5 Density and velocity contours when $M=0.8$ and $R e=50$.

$3 \%$ variation in density, whereas Fig. 6 shows a density variation of about $60 \%$.

The drag coefficient on the flat plate is calculated by integrating the skin friction coefficient on both sides of the plate [Eq. (8)], whereas the skin friction is obtained by sampling the rate of the momentum transferred from the gas molecules to the plate [Eq. (10)]:

$$
\begin{gathered}
C_{D}=\frac{1}{L} \int_{-L / 2}^{L / 2}\left(C_{f 1}+C_{f 2}\right) \mathrm{d} l \\
C_{f}=\frac{\tau_{w}}{0.5 \rho_{\infty} V_{\infty}^{2}} \\
\tau_{w}=\frac{\sum_{j} m\left(u_{j}^{\mathrm{in}}-u_{j}^{\mathrm{re}}\right)}{\Delta t \cdot \Delta A}
\end{gathered}
$$

where the subscript in Eq. (8) refers to the side of the plate. The DSMC skin friction is calculated based on the microscopic velocity whereas the IP result is based on the preserved information. Distribution of the skin friction on one side of the plate is shown in Fig. 7 when the Mach number is 0.2. The DSMC and IP results are compared when the Reynolds number is 50 and 10 . The agreement between the IP and DSMC results is very good except that the DSMC results exhibit obvious statistical scatter. However, careful comparison near the leading and trailing edges shows that there is 


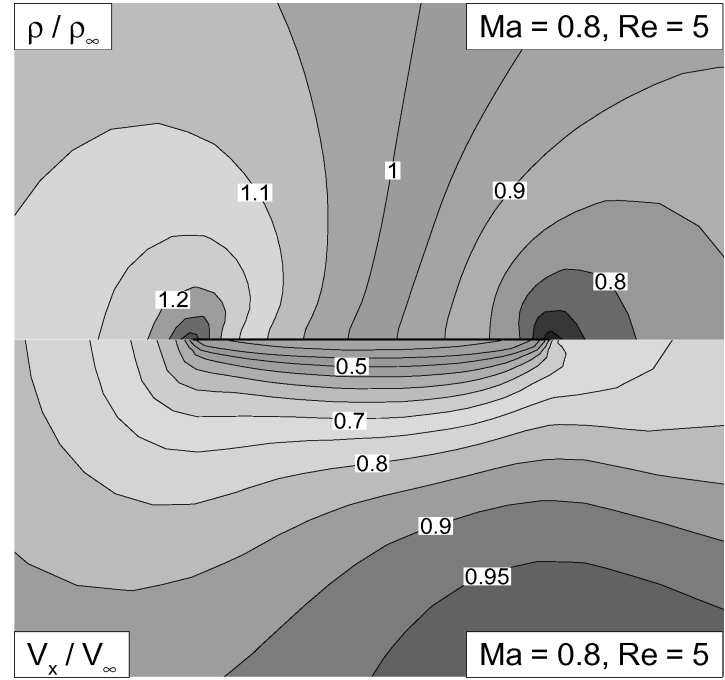

Fig. 6 Density and velocity contours when $M=0.8$ and $R e=5$.

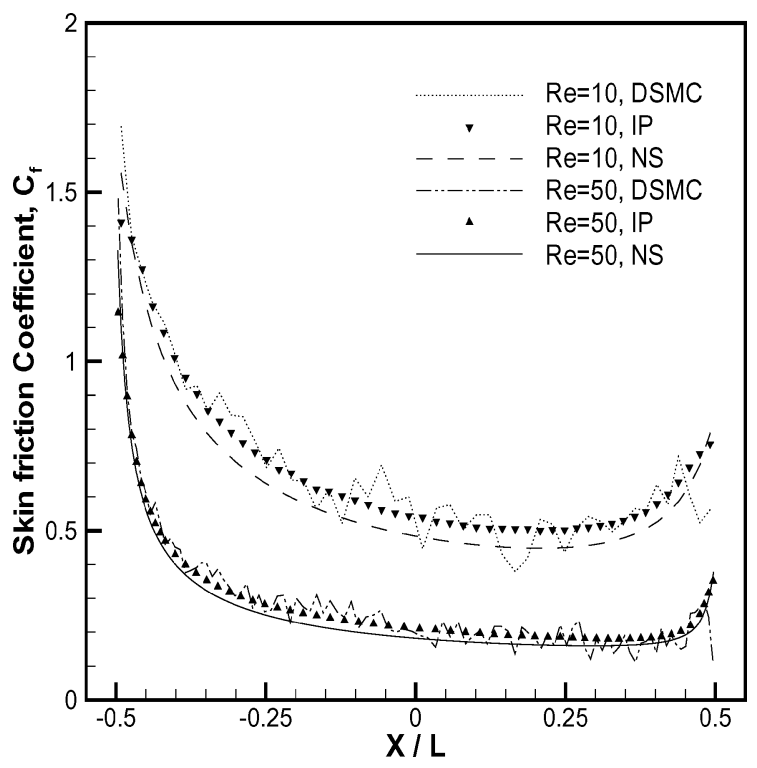

Fig. 7 Comparison of the skin friction coefficient among the IP, DSMC, and NS results when the Mach number is $\mathbf{0 . 2}$.

some difference between the DSMC and IP results. This may show that the models used in the IP method cannot capture all necessary physics in these strong nonequilibium regions. For the purpose of comparison, Fig. 7 also shows the numerical results of the NS equations using the Maxwell-type slip velocity boundary condition. ${ }^{18}$ Clearly, there are noticeable differences between the NS results and the other results, especially when the Reynolds number is small. The overall drag coefficient on the plate is plotted in Fig. 8 for a range of Reynolds numbers when the Mach number is 0.2 and 0.8 . The comparison shows that the agreement between the IP and DSMC results is good, and there is small difference when the Reynolds number is small. Again, the difference here exists because the IP method needs more physically accurate models for the strong nonequilibrium flows. However, the IP method is useful to investigate the dependence of the drag on other parameters because the scatter associated with the IP method is much less than that associated with the DSMC method.

The drag coefficient on the plate under different Mach and Reynolds number conditions is plotted in Fig. 9 for the IP results. In this figure, the solid line shows the drag coefficient obtained by Dennis and Dunwoody ${ }^{6}$ through solving the incompressible NS equations, which corresponds to the results when the Mach number is approaching zero. The symbols show the IP results when the

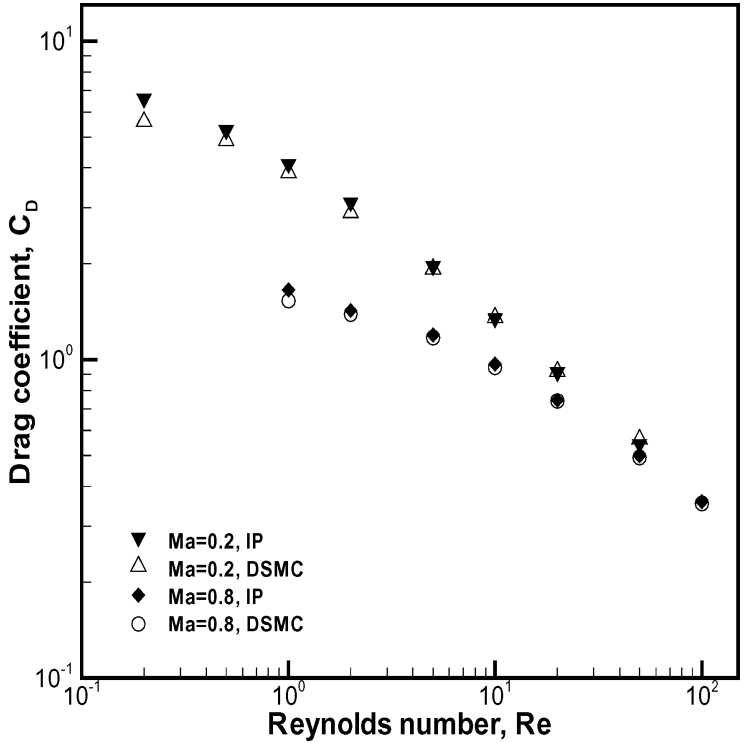

Fig. 8 Comparison of the drag coefficient between the IP and DSMC results when the Mach number is 0.2 and 0.8 .

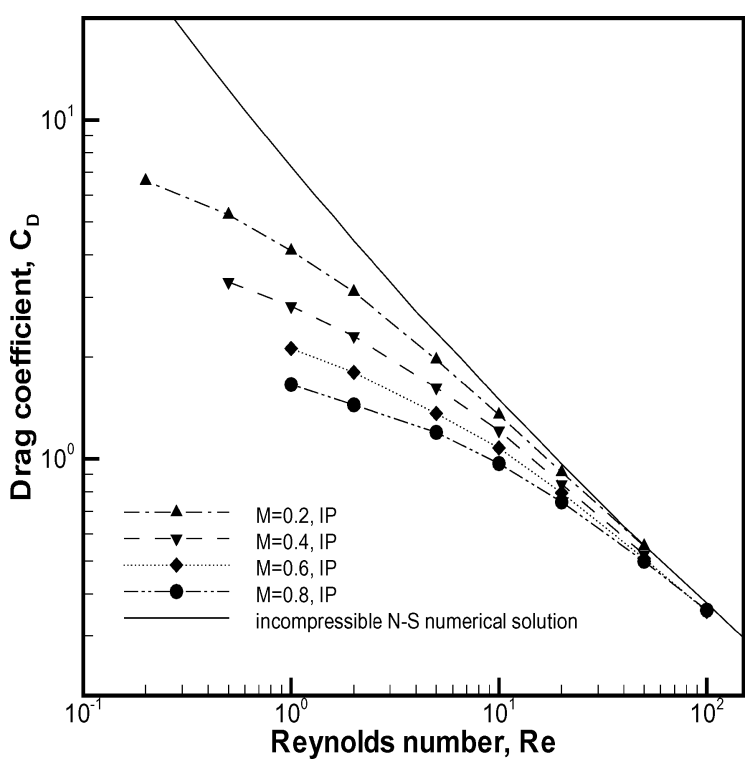

Fig. 9 Drag on the flat plate depends on Reynolds number and Mach number as predicted by the IP method.

freestream Mach number varies from 0.2 to 0.8 . It is clear that the drag coefficient on the plate decreases when the flow Mach number increases. This may indicate a compressible effect on the drag coefficient. However, the Mach number is directly related to the Knudsen number through the Reynolds number. The rarefied effects (e.g., velocity slip) may play a more important role in the drag coefficient because the compressible effect is very small at largeReynolds-number (or small-Knudsen-number) flows.

It is clear that the drag coefficient depends not only on the Reynolds number but also on the Mach number. Hence, it is useful to develop a scaling law for the drag coefficient, which will facilitate comparisons between simulated results and experimental data and be useful for engineering analysis. Schaaf ${ }^{2}$ derived a relationship [Eq. (11)] between the normalized drag coefficient $\left(C_{D} \cdot M\right)$ and a nondimensional parameter $[\sqrt{ }(R e) / M]$ by transforming a Rayleigh flow problem:

$$
\begin{gathered}
C_{D} M=\left(2.67 / z^{2}\right)\left[e^{z^{2}} \operatorname{erfc}(z)-1+2 z / \sqrt{\pi}\right] \\
z=\sqrt{\operatorname{Re}} / 1.5 M
\end{gathered}
$$


Later, Mirels ${ }^{23}$ extended the analysis to include the effects of compressibility and introduced an empirical relation to match with the Blasius solution:

$$
\begin{gathered}
C_{D} M=\left(2.697 / z^{2}\right)\left\{e^{z^{2}} \operatorname{erfc}(z)-1+2 z / \sqrt{\pi}-0.0718\right\} \\
z=1.146 \sqrt{\operatorname{Re}} / M
\end{gathered}
$$

If the relationship obtained by Schaaf or Mirels is followed, both the IP results and the experimental data obtained by Schaaf and Sherman, ${ }^{8}$ however, do not collapse well to a single curve (Fig. 10). As the almost-free-molecular theory of $\mathrm{Liu}^{24}$ shows [Eq. (13)], the normalized drag coefficient $C_{D} \cdot M$ depends on $\sqrt{ }(R e) / M$ and $G$ that is a complicated function of $M$ and $R e / M$, the present results suggest that $C_{D} \cdot M$ for the current range of $\sqrt{ }(R e) / M$ may not depend only on $\sqrt{ }(R e) / M$ :

$$
C_{D} M=1.35\left[1-0.188 \cdot(\sqrt{\operatorname{Re}} / M)^{2} G(M, \operatorname{Re} / M)\right]
$$

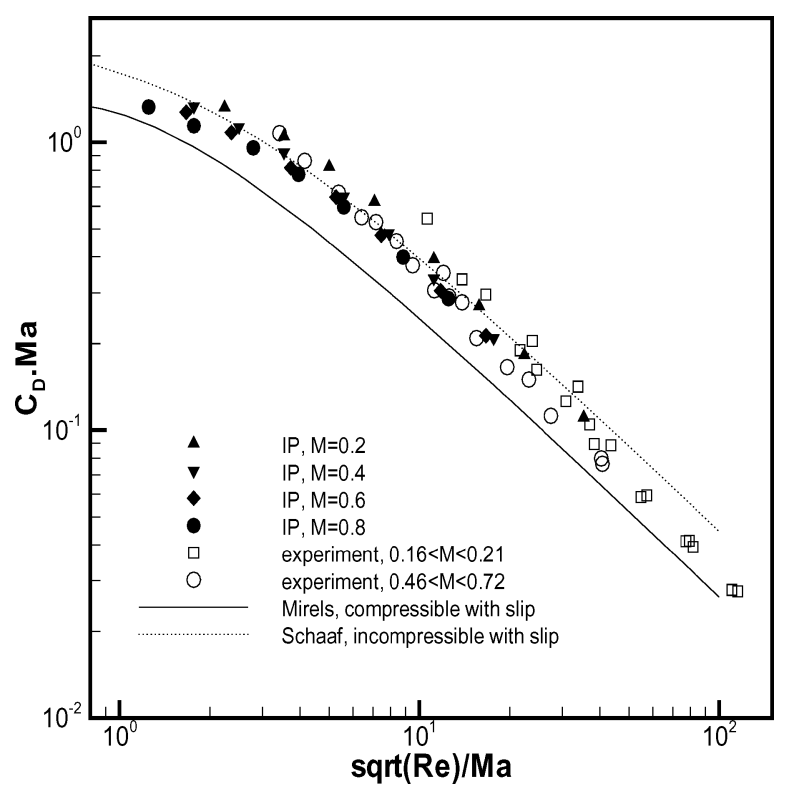

Fig. 10 Drag on the plate from the IP results and the experimental data plotted as $C_{D} \cdot M$ vs $\sqrt{ }(R e) / M$.

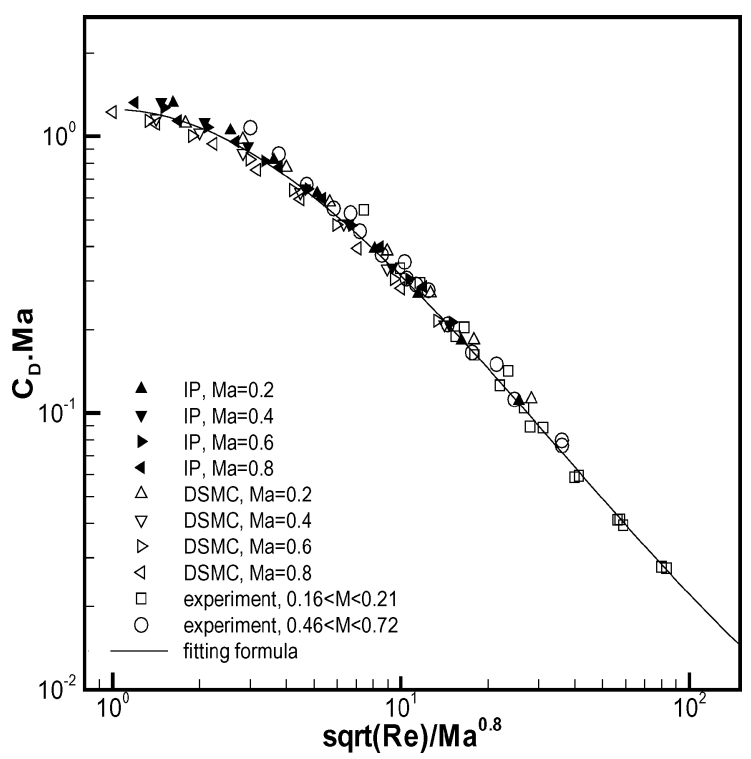

Fig. 11 Drag on the plate from the DSMC and IP results along with the experimental data plotted as $C_{D} \cdot M$ vs $\sqrt{ }(R e) / M^{0.8}$.
Careful examination of the drag in the $C_{D} \cdot M$ vs $\sqrt{ }(R e) / M$ plot shows that the profiles can be grouped with the flow Mach number. Therefore, plotting $C_{D} \cdot M$ with a variable combining $\sqrt{ }(R e) / M$ and $M$ is studied. It is found that the normalized drag coefficient $C_{D} \cdot M$ profiles correlate very well with a parameter $\sqrt{ }(R e) / M^{0.8}$ for both the simulated results and the experimental data as shown in Fig. 11. Therefore, a data-fitted scaling law for the drag coefficient on the plate is proposed as

$$
\begin{aligned}
& \ln \left(C_{D} \cdot M\right)=0.225-0.333 \times \ln ^{2}\left(\sqrt{\operatorname{Re}} / M^{0.8}\right) \\
& +0.031 \times \ln ^{3}\left(\sqrt{\operatorname{Re}} / M^{0.8}\right)
\end{aligned}
$$

when $1<\sqrt{ }(R e) / M^{0.8}<100$, which is also plotted in Fig. 11 .

\section{Conclusions}

Flow over a flat plate was investigated under subsonic, lowReynolds-number conditions. The compressible low-Reynoldsnumber flows required a kinetic approach to capture the rarefied effects. Two particle methods, namely the DSMC method and the IP method, were used to simulate the flows using characteristic boundary conditions. Good agreement was obtained between the IP results and the DSMC results and between the IP results and available experimental data. The numerical simulations predicted that the drag coefficient on the flat plate depended not only on the flow Reynolds number but also on the freestream Mach number, and both the rarefied and compressible effects increased when the flow Reynolds number decreased. Based on the simulated results and experimental data, a scaling law was proposed for the drag on the plate, which states that the normalized drag $C_{D} \cdot M$ depends on $\sqrt{ } \operatorname{Re} / M^{0.8}$ when this parameter varies between 1 and 100 .

\section{Acknowledgments}

The authors thank the Air Force Office of Scientific Research under Grant F49620-98-1-0433 for support of this work and the referees for helpful suggestions to improve the paper.

\section{References}

${ }^{1}$ Churchill, S. W., Viscous Flows: The Practical Use of the Theory, Butterworths, Stoneham, MA, 1988.

${ }^{2}$ Schaaf, S. A., "A Note on the Flat Plate Drag Coefficient," Inst. of Engineering Research, Univ. of California, Report No. HE-150-66, Berkeley, CA, 1950 .

${ }^{3}$ Laurmann, J. A., "Linearized Slip Flow past a Semi-Infinite Flat Plate," Journal of Fluid Mechanics, Vol. 11, 1961, pp. 82-96.

${ }^{4}$ Murray, J. D., "Incompressible Slip Flow past a Semi-Infinite Flat Plate," Journal of Fluid Mechanics, Vol. 22, 1965, pp. 463-469.

5 Janssen, E., "Flow past a Flat Plate at Low Reynolds Numbers," Journal of Fluid Mechanics, Vol. 3, 1958, pp. 329-343.

${ }^{6}$ Dennis, S. C., and Dunwoody, J., "The Steady Flow of a Viscous Fluid past a Flat Plate," Journal of Fluid Mechanics, Vol. 24, 1966, pp. 577-595.

${ }^{7}$ Janour, Z., "Resistance of a Plate in Parallel Flow at Low Reynolds Numbers," NACA TM 1316, 1951.

${ }^{8}$ Schaaf, S. A., and Sherman, F. S., "Skin Friction in Slip Flow," Journal of the Aeronautical Sciences, Vol. 21, 1954, pp. 85-90.

${ }^{9}$ Kogan, M. N., "Molecular Gas Dynamics," Annual Review of Fluid Mechanics, Vol. 5, 1974, pp. 383-404.

${ }^{10}$ Ohwada, T., "Structure of Normal Shock Waves: Direct Numerical Analysis of the Boltzmann Equation for Hard-Sphere Molecules," Physics of Fluids A, Vol. 5, 1993, pp. 217-234.

${ }^{11}$ Haile, J. M., Molecular Dynamics Simulation: Elementary Methods, Wiley-Interscience, New York, 1993.

${ }^{12}$ Bird, G. A., Molecular Gas Dynamics and the Direct Simulation of Gas Flows, Oxford, New York, 1994.

${ }^{13}$ Fan, J., and Shen, C., "Statistical Simulation of Low-Speed Unidirectional Flows in Transition Regime," Rarefied Gas Dynamics, Vol. 2, edited by R. Brun, R. Campargue, R. Gatignol, and J. C. Lengrand, Cepadues, Toulouse, France, 1999, pp. 245-252.

${ }^{14}$ Fan, J., and Shen, C., "Statistical Simulation of Low-Speed Rarefied Gas Flows," Journal of Computational Physics, Vol. 167, 2001, pp. 393-412.

${ }^{15}$ Sun, Q., and Boyd, I. D., "A Direct Simulation Method for Subsonic, Microscale Gas Flows," Journal of Computational Physics, Vol. 179, 2002, pp. $400-425$. 
${ }^{16}$ Cai, C., Boyd, I. D., Fan, J., and Candler, G. V., "Direct Simulation Methods for Low-Speed Microchannel Flows," Journal of Thermophysics and Heat Transfer, Vol. 14, 2000, pp. 368-378.

${ }^{17}$ Fan, J., Boyd, I. D., Cai, C., Hennighausen, K., and Candler, G. V., "Computation of Rarefied Flows Around a NACA0012 Airfoil," AIAA Journal, Vol. 39, 2001, pp. 618-625.

${ }^{18}$ Sun, Q., Boyd, I. D., and Candler, G. V., "Numerical Simulation of Gas Flow over Microscale Airfoils," Journal of Thermophysics and Heat Transfer, Vol. 16, 2002, pp. 171-179.

${ }^{19}$ Wang W.-L., and Boyd, I. D., "A New Energy Flux Model in the DSMC-IP Method for Nonequilibrium Flows," AIAA Paper 2003-3774, June 2003.

${ }^{20}$ Nance, R. P., Hash, D. B., and Hassan, H. A., "Role of Boundary Conditions in Monte Carlo Simulation of MEMS Devices," Journal of
Thermophysics and Heat Transfer, Vol. 12, 1998, pp. 447-449.

${ }^{21}$ Sun, Q., Boyd, I. D., and Candler, G. V., "A Hybrid Continuum/Particle Approach for Modeling Subsonic, Rarefied Gas Flows," Journal of Computational Physics, Vol. 194, 2004, pp. 256-277.

${ }^{22}$ Hirsch, C., Numerical Computation of Internal and External Flows-II, Wiley, West Sussex, England, U.K., 1991.

${ }^{23}$ Mirels, H., "Estimate of Slip Effect on Compressible LaminarBoundary-Layer Skin Friction,” NACA TN 2609, 1951.

${ }^{24} \mathrm{Liu}$, V. C., "On the Drag of a Flat Plate at Zero Incidence in Almost-Free-Molecular Flow," Journal of Fluid Mechanics, Vol. 5, 1959, pp. 481-490.

G. Candler Associate Editor

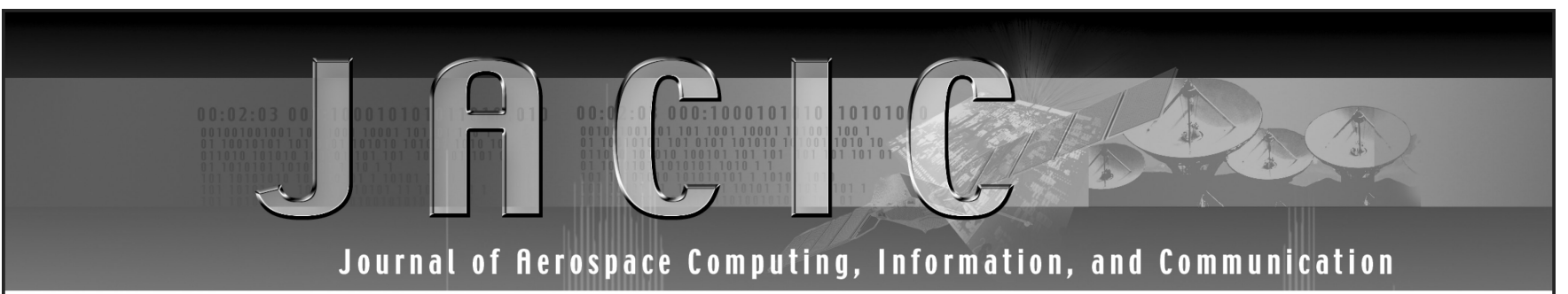

Editor-in-Chief: Lyle N. Long, Pennsylvania State University

AIAA is launching a new professional journal, the Journal of Aerospace Computing, Information, and Communication, to help you keep pace with the remarkable rate of change taking place in aerospace. And it's available in an Internet-based format as timely and interactive as the developments it addresses.

\section{Scope:}

This journal is devoted to the applied science and engineering of aerospace computing, information, and communication. Original archival research papers are sought which include significant scientific and technical knowledge and concepts. The journal publishes qualified papers in areas such as real-time systems, computational techniques, embedded systems, communication systems, networking, software engineering, software reliability, systems engineering, signal processing, data fusion, computer architecture, high-performance computing systems and software, expert systems, sensor systems, intelligent sys- tems, and human-computer interfaces. Articles are sought which demonstrate the application of recent research in computing, information, and communications technology to a wide range of practical aerospace engineering problems.

Individuals: \$40 • Institutions: \$380

$\uparrow$ To find out more about publishing in or subscribing to this exciting new journal, visit www.aiaa.org/jacic, or e-mail JACIC@aiaa.org.

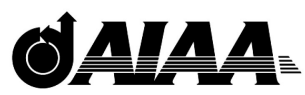

American Institute of Aeronautics and Astronautics 